\title{
Central Exclusive Production of Hadrons in CDF
}

\author{
M.G. Albrow \\ Fermi National Accelerator Laboratory, Batavia, IL 60510, USA \\ (On behalf of the CDF Collaboration, with co-authors D.Lontkovskyi and I.Makarenko (Kyiv), \\ and A.Swiech and M.Zurek (Krakow))
}

\begin{abstract}
At the Fermilab Tevatron with $\sqrt{s}=900$ and $1960 \mathrm{GeV}$, we have studied exclusive double pomeron exchange in the Collider Detector at Fermilab, CDF. With 300,000 events we present the mass spectrum of two hadrons, $h^{+} h^{-}$, assumed to be pions, with $|\eta(\pi)|<1.3$ and two rapidity gaps $\Delta \eta>4.6$. The mass spectrum shows resonance structures, including $f_{0}(980), f_{2}(1270)$, and $f_{0}(1370)$. The cross section ratio $1960 \mathrm{GeV} / 900 \mathrm{GeV}$ and the mean $p_{T}$ (pair) show massdependent structures, even above $M=2 \mathrm{GeV}$ where there are no established $\pi^{+} \pi^{-}$resonances. The data extend above $M=5 \mathrm{GeV}$. We place an upper limit on exclusive $\chi_{c 0} \rightarrow \pi^{+} \pi^{-}$and $K^{+} K^{-}$.
\end{abstract}

Keywords: Diffraction, Double pomeron exchange, Central exclusive production PACS: 12.38.Qk,13.20.Gd,13.60.-r

Central exclusive particle production events [1] in $p p(p \bar{p})^{1}$ collisions are of the type $p+p \rightarrow p(*)+X+p(*)$, where " + " means a large ( $\gtrsim 3$ units) rapidity gap devoid of hadrons, and $X$ is fully measured. The protons may emerge intact with Feynman $x_{F} \gtrsim 0.99$, or be excited to a low-mass state, $p *$. In CDF we observed, for the first time in hadron-hadron collisions: $\gamma \gamma \rightarrow e^{+} e^{-}, \mu^{+} \mu^{-}$(QED), $\gamma \mathbb{P} \rightarrow J / \psi, \psi(2 S)$ (photoproduction), and IPIP $\rightarrow \chi_{c}$ [2], $\gamma \gamma$ [3] and Jet + Jet [4](double pomeron exchange, DIPE), where $\mathrm{IP}$ denotes a pomeron. We need not define the pomeron here; let us just say it is a strongly-interacting color-singlet $t$-channel exchange, mostly gluons, that has a Regge intercept $\alpha_{\mathbb{P}}(t=0)>1$ and so, like the photon, can be exchanged over a large rapidity gap. In events of the type $p(*)+(X=$ hadrons $)+p(*)$, DIPE dominates, as $\alpha_{S}>>\alpha_{E M}$, and only states with $I^{G} J^{P C}=0^{+}(\text {even })^{++}$are allowed. This channel is therefore excellent for meson spectroscopy, especially of the important but poorly understood scalars. To quote from the Particle Data Group [5]: "The scalar mesons are especially important to understand because they have the same quantum numbers as the vacuum $\left(J^{P C}=0^{++}\right)$. Therefore they can condense into the vacuum and break a symmetry such as a global chiral $U\left(N_{f}\right) \times U\left(N_{f}\right)$. The details of how this symmetry breaking is implemented in Nature is one of the most profound problems in particle physics. In contrast to the vector and tensor mesons, the identification of the scalar mesons is a long-standing puzzle."

Above $1.5 \mathrm{GeV}$ little is known about states with $\pi \pi$ couplings, and nothing above $2 \mathrm{GeV}$, so any structures there may indicate new states. Note also that the pomeron trajectory $\alpha_{\mathbb{P}}(t) \sim 1.08+0.20 t$ has $\alpha(t) \equiv J=2$ at $t=4.6 \mathrm{GeV}^{2}$ or $M=2.14 \mathrm{GeV}$, and it has been suggested that a spin 2 glueball could be nearby. (The lightest glueball should be a scalar, and so can not be on the pomeron trajectory.) The only well-established states

\footnotetext{
${ }^{1}$ We use $p$ to mean $p$ or $\bar{p}$.
} 
with allowed DIPE quantum numbers above $2 \mathrm{GeV}$ are $\chi_{c 0,2}, \chi_{b 0,2}$ and now the Higgs boson (also allowed in exclusive production, $p+H+p$ ).

We used the Collider Detector at Fermilab, CDF, triggering on two central calorimeter towers $(|\eta|<1.3)$ with at least $0.5 \mathrm{GeV}$ energy (a very low threshold) with no energy in beam shower counters (BSC1), $|\eta|=5.4-5.9$, and in the Forward Plug calorimeter $|\eta|=2.11-3.64$. Noise levels were established with "zero-bias" bunch crossing events with no tracks. The probability that the whole detector, covering $-5.9<\eta<5.9$, is empty, as a function of bunch luminosity, is an exponential with intercept 1.0 and a slope corresponding to that part of the inelastic cross section in the covered range. In the triggered data we required the whole detector to be empty except for two oppositelycharged tracks and their corresponding calorimeter hits. (We also selected 4-track events to measure $K_{S}^{0} K_{S}^{0}$.) Because of the gap requirements in the trigger, only no-pileup beam crossings were used. We recorded $90(22) \times 10^{6}$ events at $\sqrt{s}=1960(900) \mathrm{GeV}$. The 900 $\mathrm{GeV}$ data came in a special run of 38 hours before the Tevatron was shut down, requested largely for this physics. The effective (no pile-up) luminosity was about $1.2(0.06) \mathrm{pb}^{-1}$ respectively. After requiring two well-measured oppositely-charged tracks with impact parameter $<0.1 \mathrm{~mm}$ from the beam line, and within $1 \mathrm{~cm}$ in $z$ at that point, we had $\sim 350,000$ (7595) $h^{+} h^{-}$events at $1960(900) \mathrm{GeV}$, with a raw (uncorrected) mass spectrum, assuming the hadrons are pions, shown in Fig.1 (left). We are presently studying the small "contamination" by kaons using $d E / d x$ ionization at low momenta, and $K_{S}^{0} \rightarrow \pi^{+} \pi^{-}$. There is a sharp rise at threshold, $M=2 m_{\pi}$, and a small peak at about $380 \mathrm{MeV}$ which is the $\phi(1020)$ reflection (assigning kaons the pion mass). Exclusive $\phi$ production is allowed by photoproduction, but these events may include non-exclusive background. A few $K_{S}^{0}$ are seen, which must be non-exclusive background. Notice that the $\rho(770)$ is almost absent. It is allowed by photoproduction, but with a much smaller cross section, or by Regge $\left(\rho^{*}\right)$ exchange, which should also be very small compared to IP exchange over such large gaps. The $f_{0}(980)$, the lightest narrow isoscalar resonance, is clear, but the lighter $f_{0}(600)$, or $\sigma$, is very broad (400 - 1200) MeV [5], and is not evident in Fig.1. However the acceptance is very low, and is zero for $p_{T} \lesssim 500 \mathrm{MeV} / \mathrm{c}$, in this mass region. The spectrum is dominated by a large asymmetric peak between 1.0 and $1.5 \mathrm{GeV}$. Pending a phase-shift analysis we interpret this as the $f_{2}(1270)$ and the $f_{0}(1370)$. There is a break, or change of slope, at about $1.5 \mathrm{GeV}$, which was apparent in the ISR data [6] at $\sqrt{s}=63 \mathrm{GeV}$. There seem to be structures above $1.5 \mathrm{GeV}$, to which we shall return.

The $\sqrt{s}=900 \mathrm{GeV}$ data have almost the same efficiencies and acceptance, the main difference being that the $z_{\text {vertex }}$ distribution is wider, as no low- $\beta$ condition was imposed. The mass distribution is very similar to that in Fig.1, but there is a factor $\times 20$ less total luminosity at $900 \mathrm{GeV}$. Fig.2(left) shows the ratio of the number of candidate events at $\sqrt{s}=1960 \mathrm{GeV}$ to that at $900 \mathrm{GeV}$ as a function of mass (always assuming $m_{\pi}$ ). The structures show that the $\sqrt{s}$ dependence is different in different mass regions. At $M\left(\pi^{+} \pi^{-}\right) \gtrsim 2 \mathrm{GeV}$ the ratio is similar to the ratio of luminosities, while below $1 \mathrm{GeV}$ the ratio is a factor 2-3 higher. The steep drop at $1 \mathrm{GeV}$, and the structures up to $2 \mathrm{GeV}$ suggest an interplay (or interference) of different spin states, with $J=0$ giving way to $J$ $=2$ at the $f_{2}(1370)$.

We corrected the data for acceptance and efficiency, including the trigger, using 

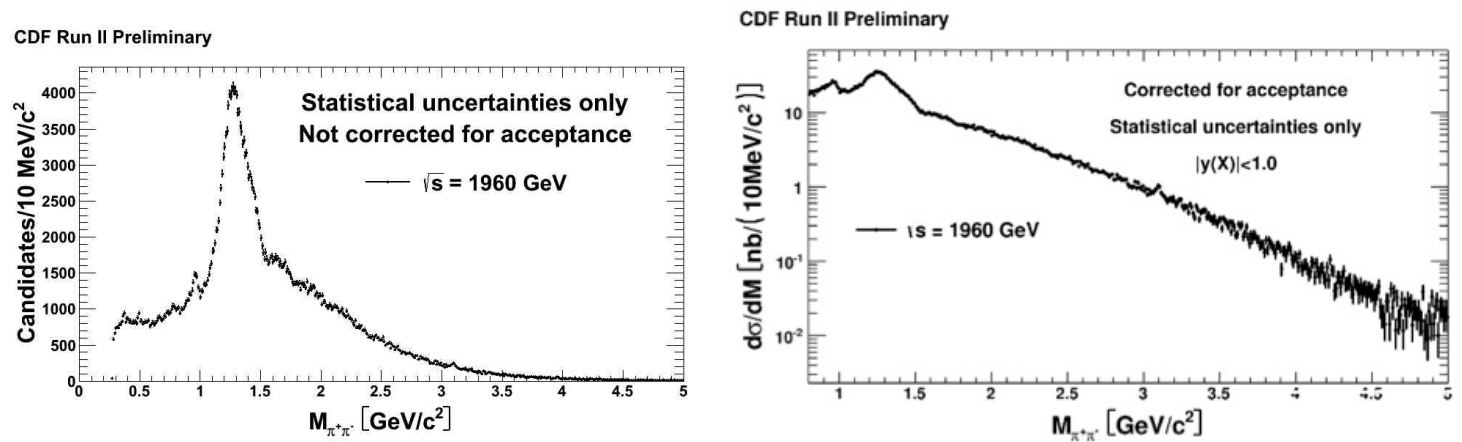

FIGURE 1. Left: Mass distribution of candidate events (not corrected for acceptance) at $\sqrt{s}=1960$ GeV. Right: Cross section for $|y|<1.0$ with two rapidity gaps $\Delta \eta=1.2-5.9$.

a simulation of the $\mathrm{CDF}$ detector. We generated the pion pair uniform in rapidity $y$ from -1 to +1 , flat in $p_{T}$ from 0 to $2.5 \mathrm{GeV} / \mathrm{c}$, and flat in mass from $2 m_{\pi}$ to 5 $\mathrm{GeV}$. After reconstruction we required $p_{T}$ (track) $>0.3 \mathrm{GeV} / \mathrm{c}$ and $\mid \eta$ (track) $\mid<1.3$, as required in the data. The acceptance does not factorize (see the plots in Ref.[7]); indeed there is no acceptance when $M(X)$ and $p_{T}(X)$ are both small. We will therefore only present cross sections, integrated over $p_{T}$, for $M\left(\pi^{+} \pi^{-}\right)>0.8 \mathrm{GeV}$, where there is acceptance at all $p_{T}$. Figure 1(right) shows the corrected data on a log scale out to 5 $\mathrm{GeV}$. Note the small peak at $3.1 \mathrm{GeV}$, near the $J / \psi$ mass. The integrated cross sections for $1.0<M\left(\pi^{+} \pi^{-}\right)<5.0 \mathrm{GeV},\left|y\left(\pi^{+} \pi^{-}\right)\right|<1.0$ is $[1910 \pm 4$ (stat) \pm 380 (syst)] nb at $1960 \mathrm{GeV}$ and $[825 \pm 11$ (stat) \pm 160 (syst) $] \mathrm{nb}$ at $900 \mathrm{GeV}$. The higher cross section at $1960 \mathrm{GeV}$ is probably due to our fixed $|\eta(\max )|$ cut at 5.9, while the beam rapidities are at $y_{\text {beam }}=\ln \left(\sqrt{\mathrm{s}} / \mathrm{m}_{\mathrm{p}}\right)=7.64$ and 6.87 respectively, allowing higher excitation masses at the higher energy.

Figure 2(right) shows the mean $p_{T}$ of the acceptance-corrected data at $1960 \mathrm{GeV}$ (the lower statistics data at $900 \mathrm{GeV}$ are consistent). While the acceptance and efficiency do not have structures, the data do, most strikingly at $1.5 \mathrm{GeV}$ where the spectra show a break, but also around $2.25 \mathrm{GeV}$ and between $3 \mathrm{GeV}$ and $4 \mathrm{GeV}$. The bin at $3.1 \mathrm{GeV}$ also has slightly higher $\left\langle p_{T}\right\rangle$.
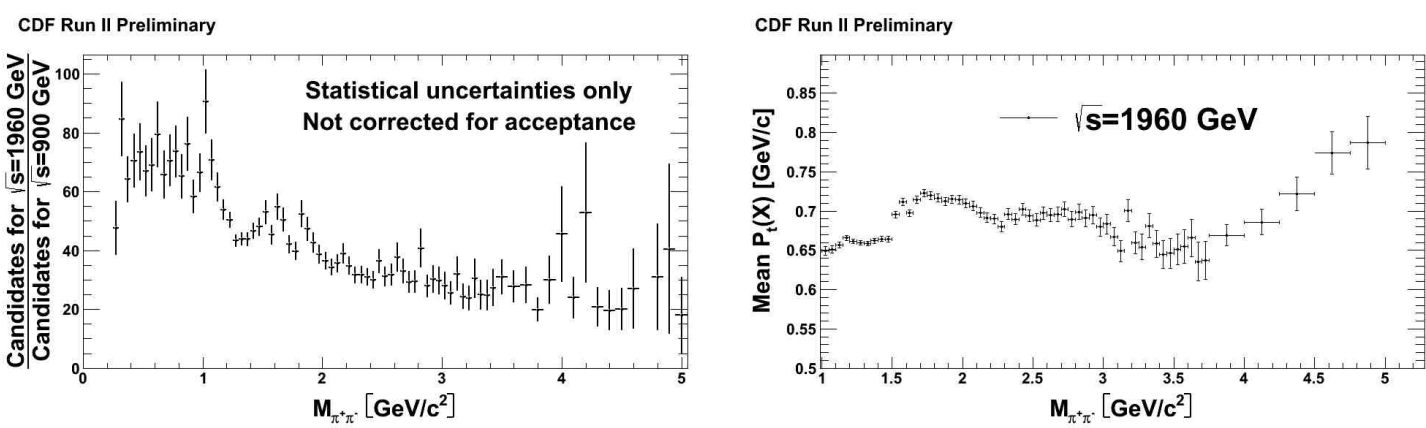

FIGURE 2. Left: Ratio of candidates at $\sqrt{s}=1960: 900 \mathrm{GeV}$ vs $M$ (pair). Right: Mean $p_{T}$ (pair) $(\mathrm{GeV} / \mathrm{c})$ as a function of $M($ pair $)$. 
In the region above $1.5 \mathrm{GeV}$ the data (Fig.1) show structures. Assuming that in the absence of resonances the spectrum should be smooth, we fit that region to a 4th-order polynomial, which gives a good overall fit, but the residuals show significant deviations up to about $2.5 \mathrm{GeV}$ [7]. We expect that a phase-shift analysis will clarify the situation.

$\mathrm{CDF}$ already published the observation of exclusive $\chi_{c}(3415) \rightarrow J / \psi+\gamma$ [2] but the photon resolution did not resolve the three $\chi_{c}$ states. If the events were all $\chi_{c 0}$ then $d \sigma / d y(y=0)=[76 \pm 10 \pm 10] \mathrm{nb}$, but if other states are present it can be much smaller, as the $J=1,2$ states have much higher branching fractions to $J / \psi+\gamma$. However the $\pi^{+} \pi^{-}$and $K^{+} K^{-}$decays not only have better mass resolution to resolve the states, but also have higher branching fractions for the $\chi_{c 0}$. We fit the mass range $2.5-5.0 \mathrm{GeV}$ with an exponential, excluding bands around the $J / \psi$ and $\chi_{c 0}$, as well as $\chi_{c 0} \rightarrow K^{+} K^{-}$ with the tracks falsely assigned the pion mass. It is a good fit, but in the excluded bands there is a clear $(4.5 \sigma) J / \psi$ peak due to $J / \psi \rightarrow e^{+} e^{-}$(we excluded events with muon candidates). This can be seen in Fig. 1 . In the two "blinded" $\chi_{c 0}$ regions we see small but not significant excesses, and put $90 \%$ C.L. upper limits on $d \sigma / d y(y=0)$ for exclusive $\chi_{c 0}$ production of $21.4 \pm 4.2$ (syst) $\mathrm{nb}$ and $18.9 \pm 3.8$ (syst) $\mathrm{nb}$ using the $\pi^{+} \pi^{-}$and $K^{+} K^{-}$ modes respectively. We conclude that our earlier observation [2] had a large contribution from $\chi_{c 1}$ and $\chi_{c 2}$.

In conclusion we have the largest sample at a hadron collider of $p+p \rightarrow p+\pi^{+} \pi^{-}+p$ data, where + are rapidity gaps $\Delta y>4.6$. The mass spectrum and its $\sqrt{s}$-dependence (and $\left\langle p_{T}\right\rangle$ ) show several structures, some of which correspond to well established isoscalar $\mathrm{J}=0,2$ resonances and others do not; these extend above $2 \mathrm{GeV}$ into a region with no known states, but where a tensor glueball is expected. We plan to do a phase-shift analysis to separate different spin states, and to study other channels, including $K_{s}^{0} K_{s}^{0}, \pi^{0} \pi^{0}, \phi \phi$ and $\eta^{0} \eta^{0}$.

\section{ACKNOWLEDGMENTS}

We thank the organizers of Diffraction 2012 for the opportunity to speak at a most enjoyable and useful meeting, and V.A.Khoze and M.G.Ryskin for discussions. We thank Fermilab and the U.S. Dept. of Energy for support.

\section{REFERENCES}

1. See M.G.Albrow, T.D.Coughlin, and J.R.Forshaw, Prog.Part.Nucl.Phys. 65, 149 (2010) for a review.

2. T.Aaltonen et al., CDF Collaboration), Phys.Rev.Lett 102, 242001 (2009).

3. T.Aaltonen et al., CDF Collaboration), Phys.Rev.Lett 108, 081801 (2012).

4. T.Aaltonen et al., CDF Collaboration), Phys.Rev. D 77, 052004 (2008).

5. J.Beringer et al., Particle Data Group, Phys. Rev. D 86, 010001 (2012).

6. T.Åkesson et al., Axial Field Spectrometer Collaboration, Nucl. Phys. B 264, 154 (1986).

7. M.G.Albrow, Central Exclusive Production in CDF at the Tevatron $p \bar{p}$ Collider, talk at this conference. 